CREAT. MATH. INFORM.

Volume 31 (2022), No. 1,

Pages 135 - 145

\title{
Exploring a Class of Substitutions and their Applications: Reflections on Teaching Foundations of Real Analysis
}

\author{
Mireya Cintora, Claudia Gutierrez, Roberto Soto, Bogdan D. Suceavă and \\ ANAEL VERDUGO
}

\section{THE MATHEMATICAL MOtivation}

In the present work we explore a technique of integration useful in calculating Riemann integrals. To better illustrate this technical idea, whose origin can be traced back to Joseph Liouville (1809 - 1882), we start our discussion with the following.

Example 1.1. For a function $f$ positive and continuous on $[0,1]$, compute:

$$
I=\int_{0}^{1} \frac{f(x)}{f(x)+f(1-x)} d x .
$$

Solution. We use a substitution that reflects the interval $[0,1]$ into itself, namely $u=$ $1-x$ which yields,

$$
I=-\int_{1}^{0} \frac{f(1-x)}{f(x)+f(1-x)} d x=\int_{0}^{1} \frac{f(1-x)}{f(x)+f(1-x)} d x
$$

The key concept is that $I$ transformed into a dual integral, and this is actually the idea originally discussed by Liouville in 1853 in Journal des Mathématiques Pures et Appliquées (not on this general example, but on a more particular case, which we show below in Figure 1). To complete the solution of our first example, note that by adding two I's together, $2 I=\int_{0}^{1} 1 d x=1$, and $I=\frac{1}{2}$. Note that the mapping $f:[0,1] \rightarrow[0,1]$ given by $f(x)=1-x$ is a bijection which "flips over" the interval $[0,1]$, and this enables the whole process.

Example 1.1 illustrates the type of $u$-substitution we plan to explore. In this fundamental example, the $u$-substitution might be rather easy to see; however, there are some cases where the approach is not evident at all.

In the comprehensive problem solving monograph [3], Section 3.2.8 is dedicated to such integration techniques. We are thinking of the present material as a complement of the existent literature on this idea, as a useful survey of relevant examples, which could serve as guide in undergraduate research seminars focused on building experience on integration techniques, or as self-study guide. The examples we collect include several recent problems not available anywhere else to English readers, while two of them do not appear, as far as we are aware, anywhere else in the present literature. We actually describe below how we construct them and why.

We rely in our selection on the tradition of the monthly Gazeta matematică, a journal with continuous publication since it was established in 1895; its first decades were much rooted in the work and the seminal influence of Gheorghe Tititeica (1873-1939), a former

Received: 31.04.2017. In revised form: 31.06.2017. Accepted: 25.01.2022

2010 Mathematics Subject Classification. 97150.

Key words and phrases. integral function; $u$-substitution; linear bijection symmetrization.

Corresponding author: Bogdan Suceavă; bsuceava@fullerton.edu 
doctoral student of Gaston Darboux (1842 - 1917) [1]. This tradition still exists today in the problems Gazeta matematică publishes every month.

The takeaway from our work is that the technique illustrated by Example 1.1 can be replicated in other applications and has a natural extension towards the limiting processes, as we will showcase below in Section 8.

\section{THE HISTORICAL MOTIVATION}

Joseph Liouville remarked in 1853 there exist classes of integrals that could be transformed or reduced to other integrals. He wrote in 1853 two brief notes $[5,6]$ under the pseudonym M. Bésge for the Journal des Mathématiques Pures et Appliquées, a journal he edited for nearly 40 years. At the end of his first note he commented specifically that "toutes ces transformations d'intégrales définies n'offrent aujourd'hui que peu d'intérêt [...]" (i.e.: "all these transformations of definite integrals don't raise today but very little interest").

$$
\int_{0}^{\frac{\pi}{3}} \varphi(\sin 2 u) \cos u d u=\int_{0}^{\frac{\pi}{2}} \varphi\left(\cos ^{2} u\right) \cos u d u,
$$

la fonction $\uparrow$ n'étant assujettie qu'aux conditions ordinaires qui font qu'on peut regarder les intégrales comme des sommes d'éléments.

On vérifiera aisément la formule que nous venons d'écrire, dans le cas où $p(x)$ est développable en série suivant les puissances de $x$ : il suffira de se rappeler l'équation connue

$$
\int_{0}^{\frac{\pi}{2}} \sin ^{n} u \cos ^{n+1} u d u=\frac{1}{2^{n}} \int_{0}^{\frac{\pi}{2}} \cos ^{2 n+1} u d u .
$$

FIGURE 1. Fragment from one of the notes written by Joseph Liouville in 1853 under the pseudonym M. Bésge. It was the same year when P. L. Chebyshev submitted to Liouville's journal his important contribution [7]. Some of the classifications we encounter in our standard references today, e.g. [9], date back to that historical period.

At the time when he wrote the two notes $[5,6]$, Liouville had a long experience with the exploration of techniques of integration, see e.g. [16]. Although Joseph Liouville discovered the procedure, it was not at all clear from the beginning what could be the range of applications that the apparent "trick" might have. A variety of examples coming from different sources is perhaps the best way to build a case in favor of such a distinct technique. It was a matter of blending the fundamental algebra with the appropriate classes of substitutions that could extend the range of definite integrals we can effectively calculate. Some are approachable and definitely a textbook case. Some others require more care, as we will illustrate below.

P. L. Chebyshev published in the same year as Liouville the work [7], in which he discussed systematically the idea of classifying procedures of integration of certain classes of functions. Since then, building on the shoulder of the 19th century giants, many other references focus on techniques of integration, e.g. $[8,13,11,17,19]$. Our present note pursues this tradition and investigates in a self-contained unit a useful integration technique.

\section{THE IDEA AS ENCOUNTERED IN CALCULUS TEXTBOOKS}

At the most fundamental level, every calculus textbook discusses at some point integrals of the type $\int e^{x} \sin x d x$ or $\int e^{x} \cos x d x$ (see e.g. [9], p.117, or [14] p. 303, or [20], p. 
512). The elementary solution involves two steps of integration by parts. The idea behind the solution is the following: we aim to obtain a linear equation where the unknown is the original integral.

The idea of solving for an indefinite integral can be seen in the following fundamental example, where the main technical argument is integration by parts.

Example 3.2. Compute $\int e^{x} \cos x d x$.

Solution. We denote the given integral by $I$, then integrate by parts:

$$
\begin{aligned}
I= & \int e^{x} \cos x d x=e^{x} \cos x+\int e^{x} \sin x d x \\
& =e^{x} \cos x+e^{x} \sin x-\int e^{x} \cos x d x
\end{aligned}
$$

Thus,

$$
I=e^{x} \sin x+e^{x} \cos x-I
$$

and solving for $I$ we immediately get

$$
I=\frac{1}{2}\left(e^{x} \sin x+e^{x} \cos x\right)+\mathcal{C} .
$$

Another fundamental example is problem 1235 from the classical reference [9].

Example 3.3. Compute $\int \sin (\ln x) d x$.

\section{Solution.}

$$
\begin{gathered}
I=\int \sin (\ln x) d x=x \sin (\ln x)-\int x \cos (\ln x) \frac{1}{x} d x= \\
x \sin (\ln x)-\int \cos (\ln x) d x= \\
x \sin (\ln x)-\left[x \cos (\ln x)-\int x \sin (\ln x) \frac{1}{x} d x=\right. \\
x \sin (\ln x)-x \cos (\ln x)-I .
\end{gathered}
$$

Thus,

$$
2 I=x \sin (\ln x)-x \cos (\ln x)
$$

and

$$
I=\frac{1}{2}[x \sin (\ln x)-x \cos (\ln x)]+\mathcal{C} .
$$

\section{IN ABSTRACT SETTING}

We started our discussion with Example 1.1, which illustrated a symmetrization of the interval of integration in abstract setting. Besides "flipping over" the interval $[a, b]$ onto itself, we can imagine the same idea working on the reflection generated by the multiplication. This example is key for our description, and it comes from [15] 1.4.13. p.23. The integrand includes, instead of a specific transcendental function, an abstract $f$ observing the analytic requirements for the existence of the desired definite integral.

Example 4.4. Let $a \neq 0$ and let $f$ be a positive and continuous function on $[0,1]$. Evaluate the integral:

$$
I=\int_{a}^{1 / a} \frac{1}{x} \cdot \frac{f(x)}{f(x)+f\left(\frac{1}{x}\right)} d x .
$$


Solution: The substitution $u=\frac{1}{x}$ yields

$$
I=-\int_{1 / a}^{a} \frac{1}{x} \cdot \frac{f\left(\frac{1}{x}\right)}{f(x)+f\left(\frac{1}{x}\right)} d x=\int_{a}^{1 / a} \frac{1}{x} \cdot \frac{f\left(\frac{1}{x}\right)}{f(x)+f\left(\frac{1}{x}\right)} d x .
$$

We obtained two copies of the same integral. Hence, by adding the two $I^{\prime}$ s together we obtain that

$$
2 I=\int_{a}^{1 / a} \frac{1}{x} d x=-\ln a^{2} .
$$

Thus $I=-\ln |a|$.

\section{THE TECHNIQUE OF TRANSFORMING INTEGRALS BY SYMMETRIZATION OF THE INTERVAL AS SEEN IN THE W. L. PUTNAM COMPETITION}

It is quite interesting that certain technical ideas developed at the middle of the 19th century are incorporated today among the problems assigned in various mathematical competitions. Around the world there are mathematical cultures where integral calculus is part of the high-school olympiad curriculum (e.g. Romania, Russia et al.), while in the United States several problems where the transformation of an integral by symmetrization of the interval have been assigned in the W.L. Putnam Competition. We start our discussion with the following example.

Example 5.5. Compute $\int_{0}^{\pi / 2} \frac{\sin ^{3} x}{\sin ^{3} x+\cos ^{3} x} d x$.

In 1983, this problem was proposed in a national olympiad examination in Romania.

Solution. Using the substitution $x=\frac{\pi}{2}-t$, since $\sin \left(\frac{\pi}{2}-t\right)=\cos t$, and $\cos \left(\frac{\pi}{2}-t\right)=\sin t$, we get:

$$
I=\int_{0}^{\pi / 2} \frac{\sin ^{3} x}{\sin ^{3} x+\cos ^{3} x} d x=\int_{\pi / 2}^{0} \frac{\cos ^{3} t}{\sin ^{3} t+\cos ^{3} t}(-d t)=\int_{0}^{\pi / 2} \frac{\cos ^{3} t}{\sin ^{3} t+\cos ^{3} t} d t .
$$

If we add two copies of $I$, we get:

$$
I+I=\int_{0}^{\pi / 2} \frac{\sin ^{3} x}{\sin ^{3} x+\cos ^{3} x} d x+\int_{0}^{\pi / 2} \frac{\cos ^{3} x}{\sin ^{3} x+\cos ^{3} x} d x=\int_{0}^{\pi / 2} d x=\frac{\pi}{2} .
$$

Thus, $I=\frac{\pi}{4}$.

A similar problem appears in [21], as well as in [15] (see 1.4.1. (g), p. 21), in general form. More precisely, by using the same idea we can compute

$$
\int_{0}^{\pi / 2} \frac{\sin ^{n} x}{\sin ^{n} x+\cos ^{n} x} d x .
$$

In the previous example, the fact that $n=3$ was irrelevant. Of course, the answer is the same, $\frac{\pi}{4}$.

For the particular case when $n=2$, it's easy to see that we get $\int_{0}^{\pi / 2} \sin ^{2} x d x=\frac{\pi}{4}$.

Example 5.6. Compute $\int_{0}^{\pi / 2} \frac{d x}{1+(\tan x)^{\sqrt{2}}}$.

The principle is the same as in Example 5.5 , for $n=\sqrt{2}$. The answer is also the same, namely $\frac{\pi}{4}$. Actually, the solution presented in [2] is in the general form, denoting $\sqrt{2}$ by $r$ and doing the same substitution. Example 5.6 is problem A3 in the W. L. Putnam Competition from 1980.

We may say that the idea of these type of substitutions appeared earlier, although with higher complexity, in the W. L. Putnam competition, problem A4 from 1953. 
Example 5.7. From the identity

$$
\int_{0}^{\pi / 2} \log \sin 2 x d x=\int_{0}^{\pi / 2} \log \sin x d x+\int_{0}^{\pi / 2} \log \cos x d x+\int_{0}^{\pi / 2} \log 2 d x,
$$

deduce the value of

$$
\int_{0}^{\pi / 2} \log \sin x d x
$$

A solution is presented in [12]. The substitutions used are $x=\frac{\pi}{2}-t$ and $x=\pi-v$, then $v=2 w$, and the goal of these substitutions is to set up an elementary algebraic equation. We want to solve this equation for the unknown, which is the given integral.

Section 3.2.8 in [3] includes a few integrals that can be approached by such substitutions. Perhaps the most famous example discussed in this reference is a definite integral assigned in the 66th W.L. Putnam Competition, in 2005, namely the computation of the definite integral.

$$
\int_{0}^{1} \frac{\ln (1+x)}{1+x^{2}} d x
$$

Before approaching this question, we focus our attention on the following.

Example 5.8. [3], Problem 458. Calculate the integral

$$
I=\int_{0}^{\frac{\pi}{4}} \ln (1+\tan x) d x
$$

We get back to this integral in Section 8. The argument in the solution is the following [3], p.536. By using the substitution $t=\frac{\pi}{4}-x$, we have $d t=-d x$, then

$$
\begin{gathered}
I=\int_{\frac{\pi}{4}}^{0} \ln \left(1+\tan \left(\frac{\pi}{4}-t\right)\right)(-1) d t=\int_{0}^{\frac{\pi}{4}} \ln \left(1+\frac{1-\tan t}{1+\tan t}\right) d t= \\
=\int_{0}^{\frac{\pi}{4}} \ln \frac{2}{1+\tan t} d t=\frac{\pi}{4} \ln 2-I .
\end{gathered}
$$

This yields the algebraic step that turns this transformation into an example relevant for our discussion. Hence, $I=\frac{\pi}{8} \ln 2$. We use this numerical result further on in the next example, as well as in Section 8.

Example 5.9. [3], Problem 459. Calculate

$$
\int_{0}^{1} \frac{\ln (1+x)}{1+x^{2}} d x
$$

This is a problem written by Titu Andreescu (see [3], p.537) for the 2005 edition of the W. L. Putnam competition. This example is giving us actually a glimpse on how to generate further examples in the same class. The solution presented in [3] explains that the substitution $t=\arctan x$, quite natural, in fact, if we take into account the term $\frac{1}{1+x^{2}}$, turns this integral into Example 5.8, namely into

$$
\int_{0}^{\frac{\pi}{4}} \ln (1+\tan x) d x
$$

For the Putnam Competition contestant, this step should have been followed by a symmetrizing substitution, as shown above in Example 5.8. The answer is, of course, $\frac{\pi}{8} \ln 2$. 


\section{A NEW EXAMPLE}

A particular challenge is when either the trigonometry or the algebra make the pattern difficult to see. In fact, the "flipping over" the interval technique we hereby illustrate works only in some cases, when the algebra intertwines well with the properties of the transcendental functions matches the mirror expressions obtained by the substitutions. The next example was written specifically for the present paper, in order to construct a corresponding integral where we use the transcendental function arcsin.

Example 6.10. Evaluate the integral:

$$
I=\int_{0}^{1} \frac{\arcsin x}{\arcsin \left[x^{\frac{3}{2}}(2-x)^{\frac{1}{2}}+(1-x)^{\frac{3}{2}}(1+x)^{\frac{1}{2}}\right]} d x .
$$

Solution: Remark first that

$$
x^{\frac{3}{2}}(2-x)^{\frac{1}{2}}+(1-x)^{\frac{3}{2}}(1+x)^{\frac{1}{2}}=x \sqrt{2 x-x^{2}}+(1-x) \sqrt{1-x^{2}} .
$$

This right-hand side term can be rewritten as:

$$
x \sqrt{1-1+2 x-x^{2}}+\sqrt{1-x^{2}}(1-x)=x \sqrt{1-(1-x)^{2}}+\sqrt{1-x^{2}}(1-x) .
$$

On the other hand, by using that $\sin (a+b)=\sin a \cos b+\cos a \sin b$ :

$$
\sin (\arcsin x+\arcsin (1-x))=x \sqrt{1-(1-x)^{2}}+\sqrt{1-x^{2}}(1-x) .
$$

In consequence, the integral we have to compute becomes:

$$
I=\int_{0}^{1} \frac{\arcsin x}{\arcsin x+\arcsin (1-x)} d x
$$

Let $t=1-x, d t=-d x$. Then

$$
I=\int_{1}^{0} \frac{\arcsin (1-t)}{\arcsin t+\arcsin (1-t)}(-d t)=\int_{0}^{1} \frac{\arcsin (1-x)}{\arcsin x+\arcsin (1-x)} d x .
$$

Therefore, $2 I=\int_{0}^{1} d x$, which yields $I=\frac{1}{2}$.

In writing this example, we proceeded by reconstructing the integrand starting from the fundamental $\sin (a+b)$ formula. The complex nature of the integrand shows us that it's not that easy to control such transcendental expressions. The key point is the relation (6.1), inspired from turning Example 1.1 into a specific exercise. One can generate such example building back from such fundamental structures.

\section{FurTher EXAMPLES: THE MORE CHALLENGING SIDE}

Example 7.11. [10] Compute the integral,

$$
\int_{0}^{\frac{\pi^{2}}{4}} \frac{1}{1+\sin \sqrt{x}+\cos \sqrt{x}} d x .
$$

Solution: We rewrite our integral I as:

$$
I=\int_{0}^{\frac{\pi^{2}}{4}} \frac{2 \sqrt{x}}{1+\sin \sqrt{x}+\cos \sqrt{x}} \cdot(\sqrt{x})^{\prime} d x
$$


With a simple substitution we let $t=\sqrt{x}$, so $x=t^{2}$ and $d x=2 t d t$. In consequence, the bounds change from 0 to $\frac{\pi}{2}$. We get

$$
\int_{0}^{\frac{\pi}{2}} \frac{2 t}{1+\sin t+\cos t} d t
$$

We make another substitution and let $y=\frac{\pi}{2}-t$ and solve for $t=\frac{\pi}{2}-y$ and so $d t=-d y$, henceforth

$$
I=2 \int_{0}^{\frac{\pi}{2}} \frac{\frac{\pi}{2}-y}{1+\sin y+\cos y} d y=\pi \int_{0}^{\frac{\pi}{2}} \frac{1}{1+\sin y+\cos y} d y-\int_{0}^{\frac{\pi}{2}} \frac{y}{1+\sin y+\cos y} d y .
$$

Notice that the second integral is our original integral, so

$$
I=\pi \int_{0}^{\frac{\pi}{2}} \frac{1}{1+\sin y+\cos y} d y-I .
$$

Once again gather our I's to get

$$
\begin{aligned}
& 2 I=\pi \int_{0}^{\frac{\pi}{2}} \frac{1}{1+\sin y+\cos y} d y \\
& I=\frac{\pi}{2} \int_{0}^{\frac{\pi}{2}} \frac{1}{1+\sin y+\cos y} d y .
\end{aligned}
$$

Let $u=\tan \frac{y}{2}$, and solve for $y=2 \arctan u$.

$$
\begin{aligned}
I & =\frac{\pi}{2} \int_{0}^{1} \frac{1}{1+\frac{2 u}{1+u^{2}}+\frac{1-u^{2}}{1+u^{2}}} \cdot \frac{2 d u}{1+u^{2}}=\pi \int_{0}^{1} \frac{d u}{1+u^{2}+2 u+1-u^{2}} \\
& =\pi \int_{0}^{1} \frac{1}{2(1+u)} d u=\frac{\pi}{2} \ln 2 .
\end{aligned}
$$

We conclude our exploration with the following example, where the "solving for I" technique needs to be combined with the symmetrization of the interval of integration.

Example 7.12. [18] Compute the integral

$$
\int_{0}^{\pi}(x+1) \cdot \frac{\sin x}{2-\sin ^{2} x} d x .
$$

Solution: Let $I$ denote the following:

$$
I=\int_{0}^{\pi}(x+1) \cdot \frac{\sin x}{2-\sin ^{2} x} d x=\int_{0}^{\pi} \frac{x \sin x+\sin x}{2-\sin ^{2} x} d x .
$$

Suppose that

$$
\begin{aligned}
I & =I_{1}+I_{2}= \\
& =\int_{0}^{\pi} \frac{x \sin x}{2-\sin ^{2} x} d x+\int_{0}^{\pi} \frac{\sin x}{2-\sin ^{2} x} d x .
\end{aligned}
$$

We start with $I_{2}$ and rewrite as,

$$
\begin{aligned}
I_{2} & =\int_{0}^{\pi} \frac{\sin x}{2-\sin ^{2} x} d x=\int_{0}^{\pi} \frac{-(\cos x)^{\prime}}{1+\cos ^{2} x} d x=\arctan (\cos x)_{0}^{\pi} \\
& =-\arctan (-1)+\arctan (1)=\frac{\pi}{2} .
\end{aligned}
$$

Similarly we rewrite $I_{1}$ as, 


$$
\begin{aligned}
I_{1} & =\int_{0}^{\pi} \frac{x \sin x}{2-\sin ^{2} x} d x=-\int_{0}^{\pi} \frac{[\pi-(x+\pi)] \sin (\pi-x)}{2-\sin ^{2}(\pi-x)} d x \\
& =\pi \int_{0}^{\pi} \frac{\sin (\pi-x)}{2-\sin ^{2}(\pi-x)} d x-\int_{0}^{\pi} \frac{(\pi-x) \sin (\pi-x)}{2-\sin ^{2}(\pi-x)} d x .
\end{aligned}
$$

Let $t=\pi-x$ and $d t=-d x$, so we have

$$
=\pi \int_{0}^{\pi} \frac{\sin x}{2-\sin ^{2} x} d x-\int_{0}^{\pi} \frac{t \sin t}{2-\sin ^{2} t} d t
$$

Initially we solved for

$$
I_{2}=\int_{0}^{\pi} \frac{\sin x}{2-\sin ^{2} x} d x=\frac{\pi}{2}
$$

and we initialized $I_{1}=\int_{0}^{\pi} \frac{t \sin t}{2-\sin ^{2} t} d t$, and thus we have

$$
\begin{aligned}
I_{1} & =\pi I_{2}-I_{1} \\
2 I_{1} & =\pi I_{2} \\
I_{1} & =\frac{\pi}{2} I_{2} .
\end{aligned}
$$

Note we established the sum of our original integral to be

$$
\begin{aligned}
I & =I_{1}+I_{2} \\
& =\frac{\pi}{2} I_{2}+I_{2} \\
& =I_{2}\left(\frac{\pi}{2}+1\right) .
\end{aligned}
$$

As calculated previously, $I_{2}=\frac{\pi}{2}$, and so

$$
I=\frac{\pi}{2}\left(\frac{\pi}{2}+1\right)
$$

Example 7.13. [4] Calculate the definite integral

$$
I=\int_{0}^{\frac{\pi}{2}} \frac{\sin x}{1+\sqrt{\sin 2 x}} d x .
$$

It was actually the editorial presentation in the January 2020 issue of the Monthly that lead us to the study of [6], since this particular integral is definitely of the class of functions that retained Liouville's attention in 1853.

Solution. By using the substitution $u=\frac{\pi}{2}-x$, we have $d u=-d x$, which in turn transforms $I$ into

$$
I=-\int_{\frac{\pi}{2}}^{0} \frac{\cos u}{1+\sqrt{\sin (\pi-2 u)}} d u=\int_{0}^{\frac{\pi}{2}} \frac{\cos x}{1+\sqrt{\sin 2 x}} d x .
$$

We reached again an interesting situation in which an integral $I$ is transformed into its dual. By adding $I$ and its dual, we obtain

$$
I+I=\int_{0}^{\frac{\pi}{2}} \frac{\sin x+\cos x}{1+\sqrt{\sin 2 x}} d x .
$$


Now a second substitution is needed, of a different nature: $v=\cos x-\sin x$, and by elementary trigonometry we derive $v^{2}=1-2 \sin x \cos x=1-\sin 2 x$, and for the differential forms we have the relationship $d v=(-\sin x-\cos x) d x$. Note that this substitution $v=v(x)$ is bijective and decreasing, and thus

$$
2 I=\int_{-1}^{1} \frac{d v}{1+\sqrt{1-v^{2}}}=2 \int_{0}^{1} \frac{d v}{1+\sqrt{1-v^{2}}} .
$$

For the last step, we proceed as in any standard textbook, $v=\sin t, d v=\cos t d t$, with

$$
\begin{gathered}
I=\int_{0}^{1} \frac{d v}{1+\sqrt{1-v^{2}}}=\int_{0}^{\pi / 2} \frac{\cos t d t}{1+\cos t}= \\
=\int_{0}^{\pi / 2} \frac{1+\cos t-1}{1+\cos t} d t=\frac{\pi}{2}-\int_{0}^{\pi / 2} \frac{1}{2 \cos ^{2} \frac{t}{2}} d t=\frac{\pi}{2}-1 .
\end{gathered}
$$

We conclude this section with another example of symmetrization. We will leave a part of the work to the reader.

Example 7.14. [22]

(a) Show that $\int_{-1}^{1} \ln \left(x^{2}+x+1\right) d x=\int_{-1}^{1} \ln \left(x^{2}-x+1\right) d x$.

(b) Calculate $\int_{-1}^{1} \ln \left(x^{4}+x^{2}+1\right) d x$.

Solution. Part (a) follows immediately by transforming $x=-t$. Part (b) is possible due to the factoring

$$
x^{4}+x^{2}+1=\left(x^{2}+x+1\right)\left(x^{2}-x+1\right) .
$$

In conclusion, the integral we calculate can be written

$$
\int_{-1}^{1} \ln \left(x^{4}+x^{2}+1\right) d x=2 \int_{-1}^{1} \ln \left(x^{2}+x+1\right) d x .
$$

This last integral can be computed directly, by parts. The numerical final answer is $3 \ln 3-$ $8+\pi \sqrt{3}$. The interesting part is that the first step of the approach must be consistent to the factoring of the four degree trinomial.

\section{THIS IDEA CAN BE APPLIED TO LIMITING PROCESSES}

Some limiting processes are directly related to the calculation of definite Riemann integrals, see e.g. Exercise 1.1.14 in [15]. We present here a new example, based on one of the integrals we presented above, see Example 5.8 just to illustrate how this process works.

Example 8.15. Show that

$$
\lim _{n \rightarrow \infty} \sqrt[n]{\left(1+\tan \frac{\pi}{4 n}\right)\left(1+\tan \frac{2 \pi}{4 n}\right)\left(1+\tan \frac{2 \pi}{4 n}\right) \ldots\left(1+\tan \frac{n \pi}{4 n}\right)}=\sqrt{2} .
$$

Solution: We can rewrite the limit we investigate in the form

$$
\Delta x\left[f\left(x_{1}\right)+f\left(x_{2}\right)+\ldots+f\left(x_{n}\right)\right],
$$

so that we actually have

$$
\begin{gathered}
\lim _{n \rightarrow \infty} \frac{\pi}{4 n}\left[\ln \left(1+\tan \frac{\pi}{4 n}\right)+\ln \left(1+\tan \frac{2 \pi}{4 n}\right)+\ldots+\ln \left(1+\tan \frac{n \pi}{4 n}\right)\right]= \\
=\frac{\pi}{4} \lim _{n \rightarrow \infty} \frac{1}{n} \ln \left[\left(1+\tan \frac{\pi}{4 n}\right)\left(1+\tan \frac{2 \pi}{4 n}\right) \ldots\left(1+\tan \frac{n \pi}{4 n}\right)\right]=
\end{gathered}
$$




$$
\begin{gathered}
=\frac{\pi}{4} \lim _{n \rightarrow \infty} \ln \sqrt[n]{\left(1+\tan \frac{\pi}{4 n}\right)\left(1+\tan \frac{2 \pi}{4 n}\right) \ldots\left(1+\tan \frac{n \pi}{4 n}\right)}= \\
=\int_{0}^{\pi / 4} \ln (1+\tan x) d x=\frac{\pi}{8} \ln 2,
\end{gathered}
$$

where the last equality comes from Example 5.8. After a simplification by $\frac{\pi}{4}$ and a step in which we exponentiate, this last calculation completes the argument.

This example is particularly relevant since it shows how wide the range of applications of the aforementioned procedure is. For each of the definite integrals discussed in this note, there is a limiting process similar to the one illustrated above.

\section{CONCLUSIONS}

After presenting all these examples, our exploration leads us to the following conclusion. There exists a class of definite Riemann integrals for which the bijective mapping symmetrizing the interval of definition is useful. In order for this process to work, we need to retrieve the original integral at some point, and this allows us to complete the computation. All our previous examples illustrate this idea. The very fact that we have been able to find so many examples in the recent literature proves there exists a genuine interest for this techniques and its applications, and teaching this process as a separate integration technique seems to us as a natural idea.

The authors hope to provide the reader with a casebook of useful gems both informative and enjoyable. Additionally, the authors would like to extend their thanks to the two referees for their very thoughtful suggestions on our present work.

\section{REFERENCES}

[1] Agnew, Alfonso F.; Bobe, Alexandru; Boskoff, Wladimir G.; Suceavă, Bogdan D. Gheorghe Ţiţeica and the origins of affine differential geometry Historia Mathematica 36 (2009), no. 2, 161-170. 00

[2] Alexanderson, G. L.; Klosinski, L. F.; Larson, L. C. The William Putnam Mathematical Competition, Problems and Solutions: 1965-1984, MAA, 1985.

[3] Andreescu, T.; Gelca, R. Putnam and Beyond. Springer-Verlag, 2009.

[4] Berindeanu, Mihaela Problem 11961, American Mathematical Monthy, 125 February 2017, p. 180. Solution in 127 January 2020, pp. 82-90.

[5] Bésge, M. (Joseph Liouville) Sur une transformation d'intégrales définies. Journal de Math. Pures et Apple. 18 (1853), p. 112.

[6] Bésge, M. (Joseph Liouville) Addition à la Note sur une transformation d'intégrales définies. Journal de Math. Pures et Apple. 18 (1853), p. 168.

[7] Chebyshev, P. L. Sur l'intégration des différentielles irrationelles. Journal des Math. Pures et Apple. XVIII (1853), pp. 87-111. Oeuvres (vide supra) vol. I pp. 147-168.

[8] Davenport, J. L. On the Integration of Algebraic Functions, Springer-Verlag, 1981.

[9] Demidovich, B. (editor), Problems in Mathematical Analysis. Mir Publishers, Moscow, seventh printing, 1989.

[10] Ghenciu, R. Problem 26450. Gazeta matematică, no. 4, 2011.

[11] Hardy, G. H. Integration of Functions. Cambridge Tracts in Mathematics and Mathematical Physics, second edition, 1916.

[12] Gleason, A. M. Greenwood, R. E.; Kelly, L. M. (editors) The William Putnam Mathematical Competition, Problems and Solutions: 1938-1964 MAA, 1980.

[13] Glesser, Adam; Shade, James; Suceavă, Bogdan D. On the Inverse Curvature Problem. College Mathematical Journal 46 (2005), 207-214.

[14] Hughes-Hallet, D. et al., Calculus, third edition, John Wiley and Sons, 2002.

[15] Kaczor, W. J.; Nowak, M. T. Problems in Mathematical Analysis III. AMS, 2003.

[16] Liouville, J. Memoire sur l'integration d'une classe de fonctions transcendantes. Journal für die reine und angewandte Mathematik 13 (1835) 93-118.

[17] Marchisotto, E.; Zakeri, G. An Invitation to Integration in Finite Terms. The College Mathematics Journal 25 (1994), no. 4 295-308.

[18] Morozan, E. Problem 20303. Gazeta matematică, no. 12, 1984. 
[19] Ritt, J. F. Integration in Finite Terms: Liouville's Theory and Elementary Methods, Columbia Univ. Press, 1948.

[20] Stewart, J. Calculus, fifth edition, Thomson, Brooks/Cole, 2003.

[21] Thomas, George B. Jr.; Finney, Ross L. Calculus and Analytic Geometry (9th Edition), Addison Wesley, 1996.

[22] Vernescu, A. Problem 27500. Gazeta matematică, no. 2, 2018.

DepartMENT OF MATHEMATiCS

CALIFORNIA STATE UNIVERSiTY, FULLERTON

800 N. State College Blvd., Fullerton, CA 92834-6850

Email address: mcintoraecsu. fullerton.edu

Email address: rcsoto@fullerton.edu

Email address: bsuceava@ fullerton.edu

Email address: averdugo@fullerton.edu

DEPARTMENT OF MATHEMATICS

MOORPARK COLLEGE

7075 CAMPUS ROAD

MOORPARK, CA 93021

Email address: cgutierrez@vcccd.edu 